\title{
THE INFLUENCE OF INCREASING LIFE EXPECTANCY ON THE DYNAMICS OF SIRS SYSTEMS WITH IMMUNE BOOSTING
}

\author{
M. P. DAFILIS ${ }^{1}$, F. FRASCOLI ${ }^{2}$, J. G. WOOD ${ }^{3}$ and J. M. MCCAW ${ }^{\circledR 1}$
}

(Received 31 July, 2012; revised 15 October, 2012; first published online 9 April, 2013)

\begin{abstract}
Endemic infectious diseases constantly circulate in human populations, with prevalence fluctuating about a (theoretical and unobserved) time-independent equilibrium. For diseases for which acquired immunity is not lifelong, the classic susceptible-infectiousrecovered-susceptible (SIRS) model provides a framework within which to consider temporal trends in the observed epidemiology. However, in some cases (notably pertussis), sustained multiannual fluctuations are observed, whereas the SIRS model is characterized by damped oscillatory dynamics for all biologically meaningful choices of model parameters. We show that a model that allows for "boosting" of immunity may naturally give rise to undamped oscillatory behaviour for biologically realistic parameter choices. The life expectancy of the population is critical in determining the characteristic dynamics of the system. For life expectancies up to approximately 50 years, we find that, even with boosting, damped oscillatory dynamics persist. For increasing life expectancy, the system may sustain oscillatory dynamics, or even exhibit bistable behaviour, in which both stable point attractor and limit cycle dynamics may coexist. Our results suggest that rising life expectancy may induce changes in the characteristic dynamics of infections for which immunity is not lifelong, with potential implications for disease control strategies.
\end{abstract}

2010 Mathematics subject classification: 92D30.

Keywords and phrases: infectious disease, mathematical model, dynamical systems.

\section{Introduction}

The susceptible-infectious-recovered (SIR) model of infectious disease transmission dynamics forms the basis of the field of infectious disease modelling [1]. It describes

\footnotetext{
${ }^{1}$ Melbourne School of Population Health and Global Health \& Murdoch Childrens Research Institute, The University of Melbourne, Victoria 3010, Australia; e-mail: mdafilis@unimelb.edu.au, jamesm@unimelb.edu.au.

${ }^{2}$ Department of Mathematics and Statistics, The University of Melbourne, Victoria 3010, Australia; e-mail: federico.frascoli@unimelb.edu.au.

${ }^{3}$ School of Public Health and Community Medicine, The University of New South Wales, Sydney 2052, Australia; e-mail: james.wood@unsw.edu.au.

(C) Australian Mathematical Society 2013, Serial-fee code 1446-1811/2013\$16.00
} 
the spread of a direct person-to-person transmissible pathogen (which elicits immunizing protection) through the population. When considering the dynamics of infection over demographic timescales, we include the processes of birth and death. Furthermore, for diseases to which immunity is not lifelong, hosts return to the susceptible state after some characteristic time. The susceptible-infectiousrecovered-susceptible (SIRS) model describes this system:

$$
\begin{aligned}
& \frac{d S}{d t}=-\beta I S+\kappa R+\xi(1-S), \\
& \frac{d I}{d t}=\beta I S-\gamma I-\xi I, \\
& \frac{d R}{d t}=\gamma I-\kappa R-\xi R .
\end{aligned}
$$

The infection rate is given by $\beta$ and the duration of infectiousness by $1 / \gamma$, with $\kappa$ the immune waning rate (hosts are protected from infection for a period $1 / \kappa)$. The population size is held constant by assuming constant and equal birth and death rates, $\xi$, with life expectancy given by $1 / \xi$ and no infection-associated mortality.

The dynamics of the system over demographic timescales are well understood $[1,10]$. If $R_{0}=\beta / \gamma$ is less than 1 , the system converges to the disease-free equilibrium $\{S, I, R\}=\{1,0,0\}$. If $R_{0}>1$, the system sustains a nonzero prevalence of infection. Assume, without loss of generality, that the population is initially fully susceptible. Upon introduction of an infective individual, the prevalence of infection, $I(t)$, grows exponentially until the susceptible pool, $S(t)$, is sufficiently depleted. $I(t)$ then peaks and declines as infectives recover. As $S(t)$ is replenished through births, $\xi$, and waning of protection, $\kappa$, further "outbreaks" of infection ensue. Over time, these transients are damped and the system converges to an endemic equilibrium, $\{S(t), I(t), R(t)\}=\left\{S^{*}, I^{*}, R^{*}\right\}$ with $S^{*}=1 / R_{0}$, where each infective in the population replaces themselves.

For diseases to which immunity is not lifelong, such as pertussis, there is emerging evidence that exposure to circulating disease while at "intermediate" levels of immunity may result in a boosting of immunity [11]. This is supported by revaccination studies, where subjects with low to intermediate antibody responses almost universally show large increases in antibody levels that correlate with immune protection [4]. In the context of assessment of optimal vaccination strategies against pertussis, Lavine et al. [11] have recently performed a detailed analysis of this dynamical system in the presence of vaccination, suggesting that increasing levels of vaccination may be responsible for the recently observed resurgence of pertussis in some Western countries.

More generally, the underlying mechanisms that act to maintain oscillations in the prevalence of disease in the community have been investigated from both an empirical and model-based perspective. Bartlett [2] pointed out that stochastic effects can act to maintain oscillations in otherwise damped systems. Earn et al. [8] argued that both endogenous nonlinear feedback mechanisms and exogenous forcing effects can have 


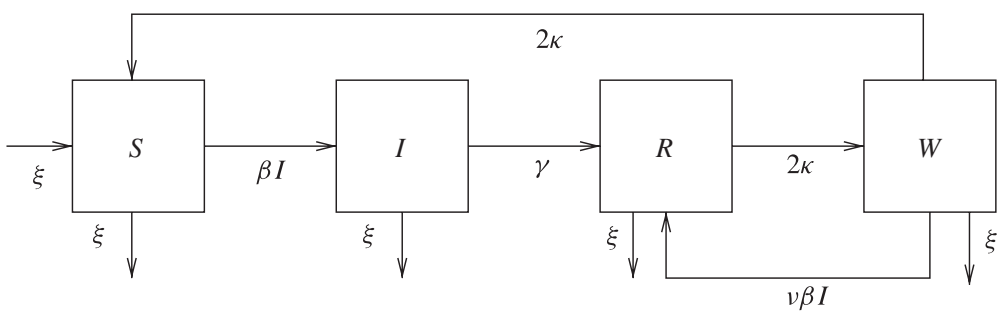

FIGURE 1. Flow diagram for the SIRWS system (2.1)-(2.4). Following infection $I$, recovered individuals $R$ return to the susceptible class $S$ through the intermediate waning state $W$. Immune boosting from $W$ to $R$ occurs due to exposure to infectious individuals.

dramatic impacts on the dynamics of transmissible diseases, generating, among other behaviours, undamped oscillatory dynamics. Bauch and Earn [3] took this principle and applied it to time series data on measles, chickenpox, rubella and whooping cough, considering both seasonal forcing and stochastic effects.

Here we explore the characteristic dynamics of the SIRS system with immune boosting in the absence of vaccination, with a particular focus on the effect of increasing life expectancy. We consider our findings in light of the changing patterns of disease transmission over the latter part of the twentieth century (at least for pathogens to which immunity is not lifelong). Our study seeks to complement other investigations into the endogenous mechanisms that drive undamped oscillatory behaviour [8].

\section{The SIRWS model with demography and immune boosting}

Our model system (Figure 1), assuming a well-mixed population following mass action dynamics, is described by the following system of equations:

$$
\begin{aligned}
\frac{d S}{d t} & =-\beta I S+2 \kappa W+\xi(1-S), \\
\frac{d I}{d t} & =\beta I S-\gamma I-\xi I, \\
\frac{d R}{d t} & =\gamma I-2 \kappa R+v \beta I W-\xi R, \\
\frac{d W}{d t} & =2 \kappa R-2 \kappa W-v \beta I W-\xi W .
\end{aligned}
$$

New infections are created at rate $\beta I S$ and the duration of infection is given by $1 /(\gamma+\xi)$, reduced from $1 / \gamma$ in a model without demography due to natural death during the infectious period. It follows that the basic reproductive number, $R_{0}$, determining the threshold for invasion and sustained nonzero prevalence of disease, is given by $R_{0}=\beta /(\gamma+\xi)$.

Boosting is modelled by introducing the intermediate waning state, $W$, whose members cannot transmit infection but may be "boosted" back to the fully protected state $R$. Boosting occurs at a rate $v$ times the force of infection, $\beta I$, as a result of 
exposure to infectious individuals. In the absence of boosting $(v=0)$, the timescale for returning to the susceptible class following infection is given by $2 /(2 \kappa+\xi)$, which in the absence of demography is $1 / \kappa$ as for the SIRS model without the intermediate state $W$.

In many existing models that consider immune boosting (see the paper by Lavine et al. [11] for more details), it is assumed to occur at the same rate as or a lower rate than infection, that is, $v$ is taken to be in $[0,1]$. However, as argued by Lavine et al. [11], it is immunologically plausible that boosting may occur through exposures that would be insufficient to cause infection in a susceptible person. Analogous to the boosting effect seen in vaccination studies [4] mentioned earlier, an individual with a primed immune system (that is, in the $W$ class) may generate a strong immunological response upon (re-)exposure to the pathogen. As such, here we consider all positive values for $v$ including in excess of 1 .

We immediately note two limiting parameterizations of the boosting model. For $v \rightarrow 0$, we expect to recover SIRS dynamics (with $R \rightarrow S$ formed as a two-stage process). For $v \rightarrow \infty$, we expect to recover SIR dynamics. We make use of well-known analytical forms for the periods of oscillation in these two cases when examining the numerics of our model system.

2.1. Equilibria and stability analysis We determine the endemic equilibrium point of our system numerically by setting the left-hand sides of equations (2.1)-(2.4) to zero and solving for the equilibrium values of $S, I, R$ and $W$ for a given set of parameters. To consider the stability of the dynamical system about this point, we must examine the eigenvalues of the Jacobian at the endemic equilibrium.

Numerical integrations of the dynamical system were performed using XPPAUT [9], using a Dormand-Prince 8(3) adaptive integrator. The timestep was fixed at 0.05 years and integrations proceeded for 2000 years to ensure that solutions had adequately converged to their final attractor. All phase planes are plotted with the first 5 years considered as an initial transient, allowing the phase plane plots to better illustrate the nature of the convergence of the dynamics onto the attractor.

Unless otherwise stated, we henceforth fix the infectious duration (in the absence of death) to $1 / \gamma=1 / 17$ years (21 days) and the immune waning rate (similarly in the absence of death) to $\kappa=1 / 10$ years $^{-1}$. We set $\beta=260$, which gives $R_{0}=15.2$. We work with a normalized population size such that $S+I+R+W=1$. These parameters correspond to the baseline choices used by Lavine et al. [11] in their investigation of pertussis.

2.2. The effect of changing life expectancy Figure 2 shows the representative dynamics of the system for life expectancies $(1 / \xi)$ of 50 and 80 years, with the level of boosting fixed at $v=5$. For $1 / \xi=50$ years the system displays damped oscillatory dynamics, while for $1 / \xi=80$ years it displays sustained oscillations. This indicates that not all eigenvalues of the Jacobian evaluated at the endemic equilibrium have negative real parts. Figure 3 shows the eigenvalues as a function of $v$ (left) and the range for $v$ over which the real part of the least damped pair of conjugate eigenvalues 

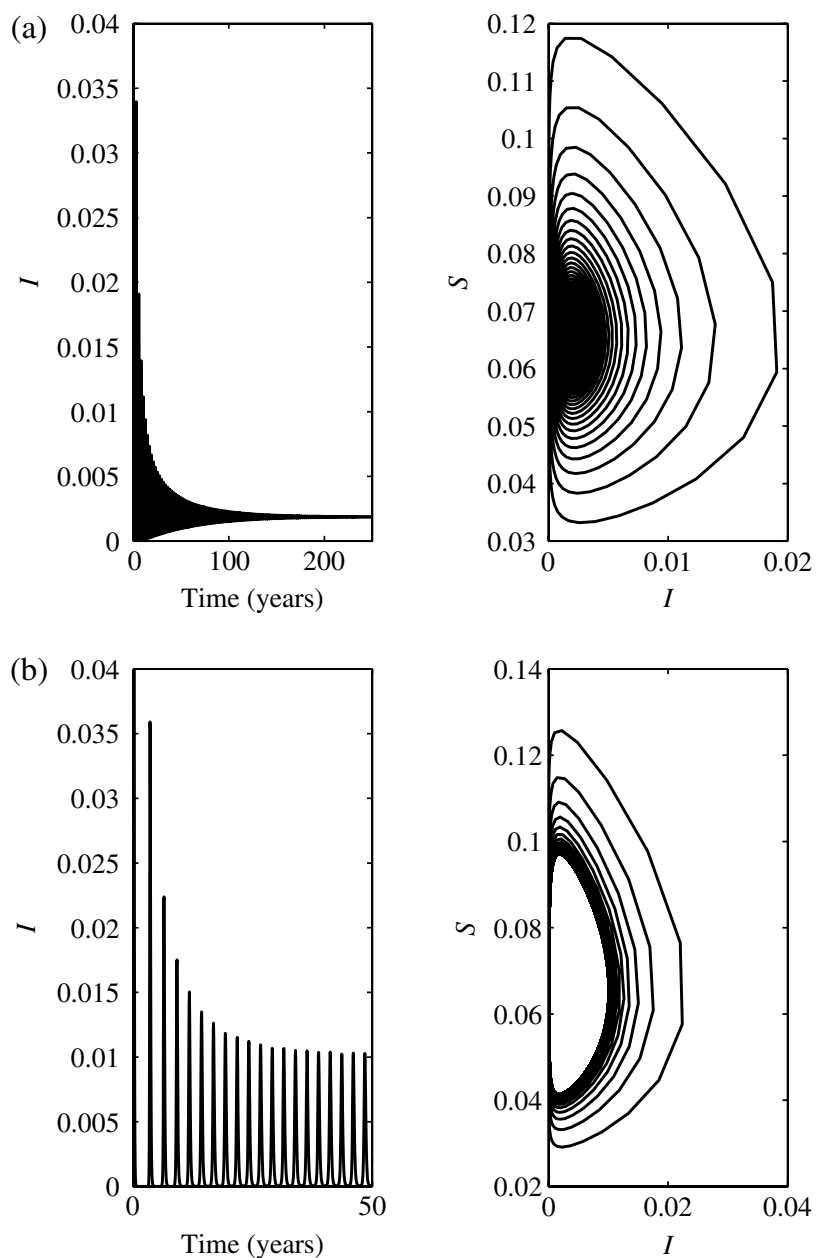

FIGURE 2. Representative time series (left) and $(S, I)$-plane phase diagrams (right) for the SIRWS model with boosting $(v=5)$, with life expectancies of (a) 50 years and (b) 80 years. The initial conditions for the system are $\{S, I, R, W\}_{0}=\{0.99,0.01,0,0\}$. For a life expectancy of 50 years, the system converges to the endemic equilibrium (point attractor). For a life expectancy of 80 years, oscillations in prevalence are sustained (stable limit cycle).

is positive (right) for life expectancies of 50 years and 80 years. The stable limit cycle, yielding undamped oscillatory dynamics, for $1 / \xi=80$ years, is confirmed.

As life expectancy increases, the system transitions from point attractor (damped oscillatory) to limit cycle (undamped oscillatory) dynamics. The characteristics of this transition are dependent on the other parameter values and, in particular, on the level of boosting, $v$. To further characterize the system, we determine one- and twoparameter bifurcation diagrams using AUTO [5-7] (Figures 4, 8 and 9) and the AUTO component of XPPAUT [9] (Figures 5 and 7). 

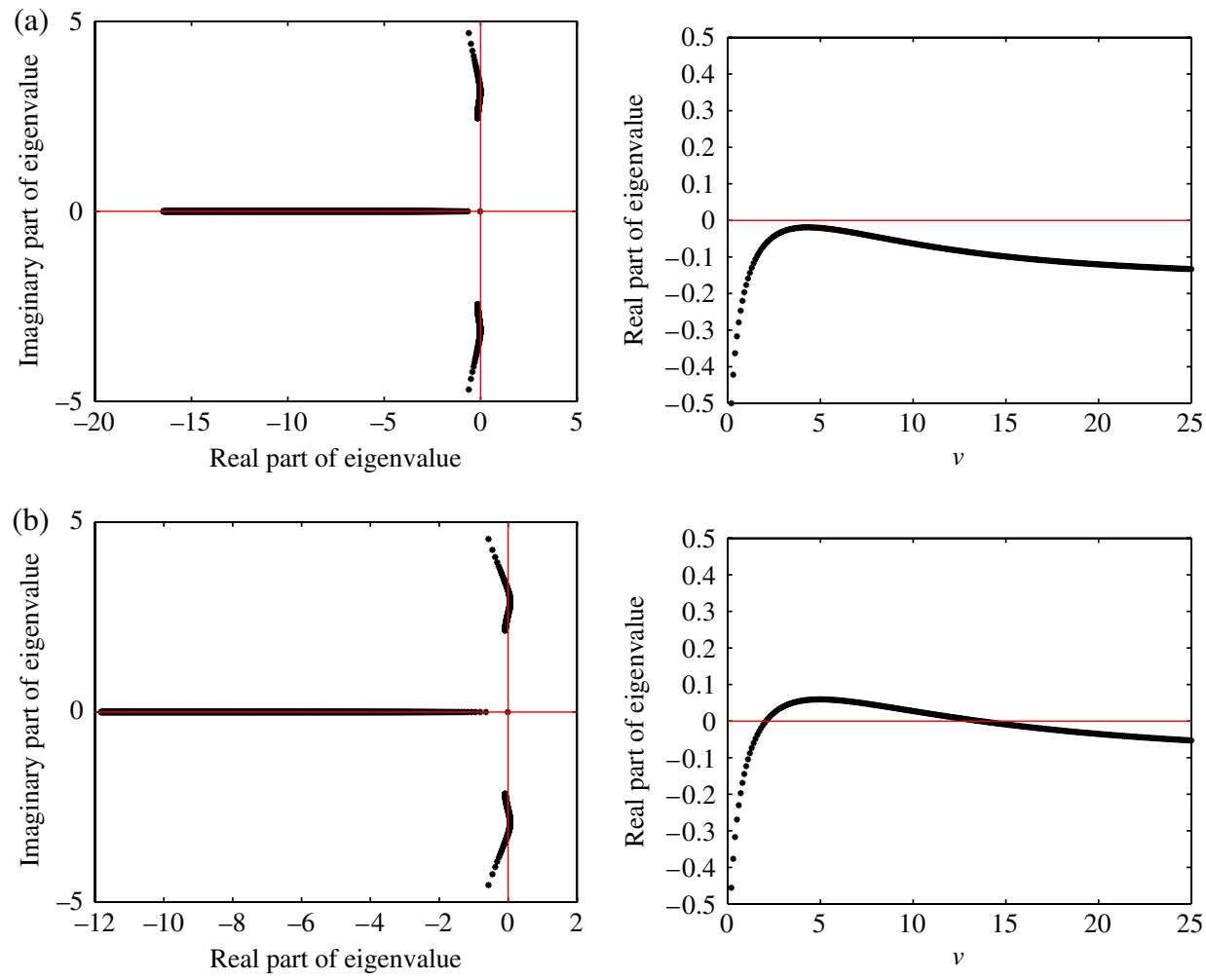

FIGURE 3. The eigenvalues of the Jacobian at the endemic equilibrium for (a) $1 / \xi=50$ years and (b) $1 / \xi=80$ years. Left: Numerical traces in the complex plane for the four eigenvalues of the Jacobian at the endemic equilibrium, as $v$ varies from 0 to 50 in steps of 0.1 . Right: The real part of the most weakly damped (conjugate pair) eigenvalue as a function of $v$. For $1 / \xi=80$, the system has positive real parts for $2 \lesssim v \lesssim 15$, indicating that sustained oscillations are present.

Figure 4 shows a two-parameter $(\xi, v)$ Hopf bifurcation diagram for the system. On the right (birth rate $\xi=0.02$ or life expectancy $1 / \xi=50$ years) the system is characterized by a stable point attractor for all values of $v$, that is, for all levels of boosting the system displays damped oscillatory dynamics. As life expectancy increases (from right to left), the system undergoes a Hopf bifurcation, picking up two supercritical Hopf lines (magenta), such that for $v$ intermediate between these values the system is characterized by a stable limit cycle (undamped oscillatory dynamics). For the chosen parameters describing the disease $(\beta, \gamma$ and $\kappa)$, this transition occurred for $1 / \xi \approx 60$ years.

The nature of the Hopf bifurcations in this region is evident by examining as an exemplar the case where $\xi=0.015$. Here both Hopf points for the system are supercritical. For values of $v$ either side of the Hopf points $\left(v<v_{\mathrm{H} 1}\right.$ or $\left.v>v_{\mathrm{H} 2}\right)$, point attractor dynamics exist and no bistability is apparent. As life expectancy increases further, the Hopf bifurcation changes from supercritical to subcritical at 


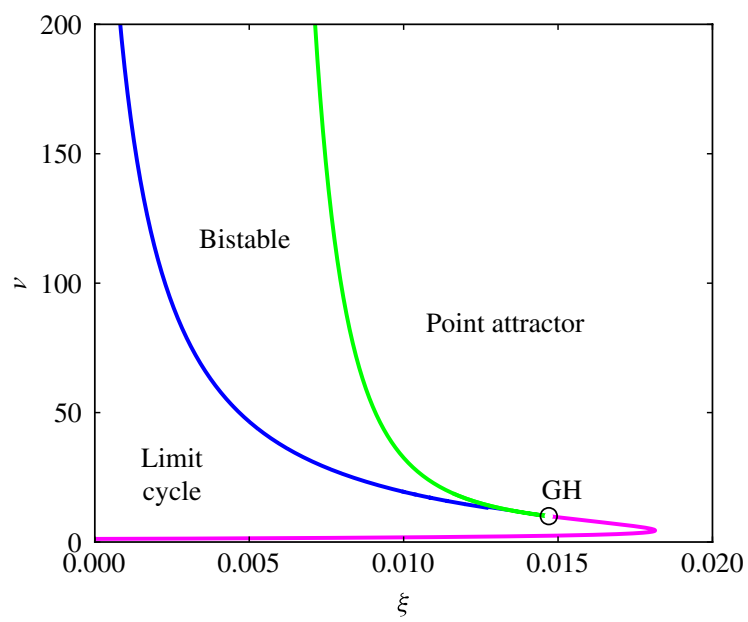

FIGURE 4. Two-parameter bifurcation diagram over birth rate $\xi$ and boosting $v$. Supercritical Hopf lines are shown in magenta, with the dynamics changing nature at point GH (open circle, a generalized Hopf (or Bautin) point), to involve a subcritical Hopf line (blue) and a limit point of periodic solutions branch (green). For high birth rates (low life expectancy) the dynamics are characterized by damped oscillatory behaviour for all values of boosting (in the neighbourhood of $\xi=0.02$ ). For longer life expectancies, the dynamics may be characterized by either point attractor or limit cycle dynamics. For $\xi<\xi_{\mathrm{GH}}$ (life expectancies greater than approximately 70 years), the dynamics may be damped, undamped or bistable depending upon the level of boosting. (Colour available online.)

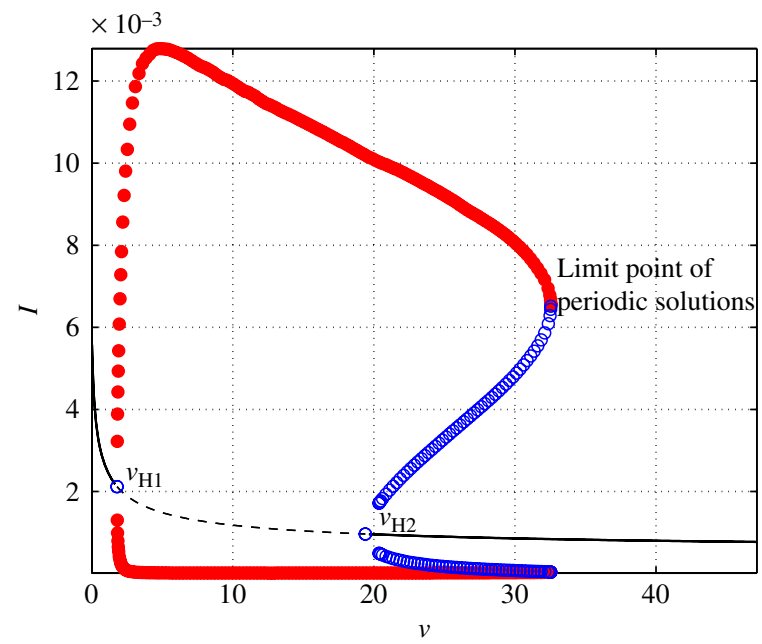

FIGURE 5. Bifurcation diagram for $v$, showing the existence of the Hopf points $v_{\mathrm{H} 1}$ and $v_{\mathrm{H} 2}$, the endemic equilibrium (black line, solid when stable, dashed when unstable) and the limit cycle (circles, solid red when stable, open blue when unstable). (Colour available online.) 
the generalized Hopf (or Bautin) point, $\xi=\xi_{\mathrm{GH}} \approx 1 / 70$ years $^{-1}$ (open circle). For $v$ between the subcritical Hopf line (blue) and the limit point of periodic solutions bifurcation line (green) $\left(v_{\mathrm{H} 2}<v<v_{\mathrm{LP}}\right)$, the system exhibits bistable behaviour (as explored in detail in Section 2.3).

This analysis demonstrates that a system with immune boosting that is naturally characterized by damped oscillatory behaviour for plausible values of all biological parameters and a life expectancy of 50 years may undergo a dramatic change as life expectancy increases beyond 60 and then 70 years.

2.3. Characterization of the system as a function of boosting We now investigate our system for a fixed life expectancy of $1 / \xi=100$ years. This value lies sufficiently to the left of the generalized Hopf point in Figure 4 such that there is a clear distinction between the subcritical Hopf and limit point of periodic solutions lines. For $1 / \xi=100$ years the characteristic dynamics may be damped, oscillatory or bistable.

Figure 5 summarizes the nature of the oscillatory dynamics of the system as a function of $v$. The vertical axis shows the maximum and minimum values for the prevalence $I$ as $t \rightarrow \infty$. The equilibrium is shown as a black line, solid when the equilibrium is stable, and dashed when it is unstable. From left to right, the first Hopf bifurcation at $v=v_{\mathrm{H} 1} \approx 2$ is supercritical and the second at $v=v_{\mathrm{H} 2} \approx 20$ is subcritical.

For $v<v_{\mathrm{H} 1}$, the equilibrium state is stable and the system is "SIRS-like", displaying damped oscillatory dynamics for all parameter choices (such that $R_{0}>1$ ). For $v_{\mathrm{H} 1}<$ $v<v_{\mathrm{H} 2}$, the equilibrium state is unstable and the system has a stable limit cycle (solid red circles). The amplitude of oscillations is largest for $v \approx 5$.

The region of stable oscillatory dynamics emanating from $v_{\mathrm{H} 1}$ extends beyond $v_{\mathrm{H} 2}$. In this region $\left(v>v_{\mathrm{H} 2}\right)$, a stable limit cycle (undamped oscillatory dynamics) coexists with a stable point attractor, leading to a region of bistability. An unstable low amplitude limit cycle also exists (open blue circles). The end point of the bistability region is the limit point of the periodic solution, at $v \approx 33$. As $v$ increases further we leave the region of bistability and the system is "SIR-like", exhibiting damped oscillatory dynamics.

2.4. Sample traces and phase space diagrams Figure 6 shows representative time series and $(S, I)$-phase plane plots for six representative values of boosting $v$ chosen from below, between and above the Hopf points identified in Figure 5. SIRS- and SIR-like dynamics are recovered for the extreme values of $v$ (Figures 6(a) and (f)). In between, we observe stable limit cycle dynamics (Figures 6(c) and (d)) and bistable mixed dynamics (Figures 6(e)).

2.5. The period of oscillations As previously noted, our system displays SIRSlike dynamics as $v \rightarrow 0$ and SIR-like dynamics as $v \rightarrow \infty$. The period of oscillations for the dynamical system is given by $2 \pi /|\mathfrak{J}(\lambda)|$, where $\lambda$ is one of the complex conjugate eigenvalues of the Jacobian evaluated at the equilibrium. Figure 7 shows the numerically determined period of oscillations as a function of $v$, picking up the values for both the stable (solid red circles) and unstable (open blue circles) limit cycles in the region of bistability. 

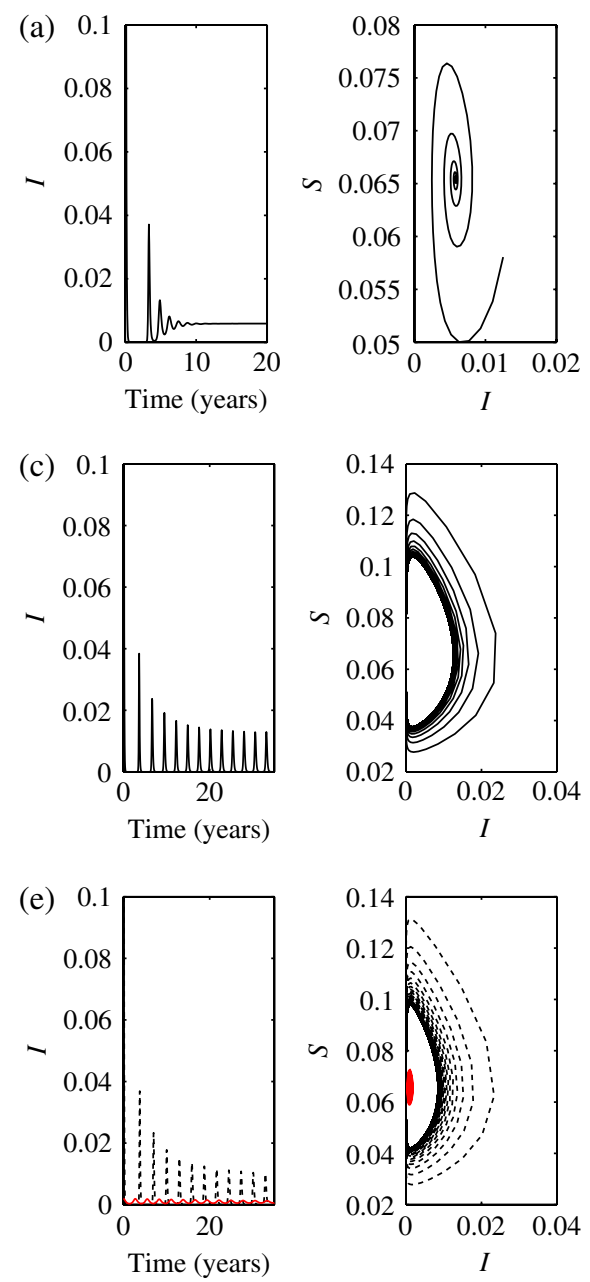
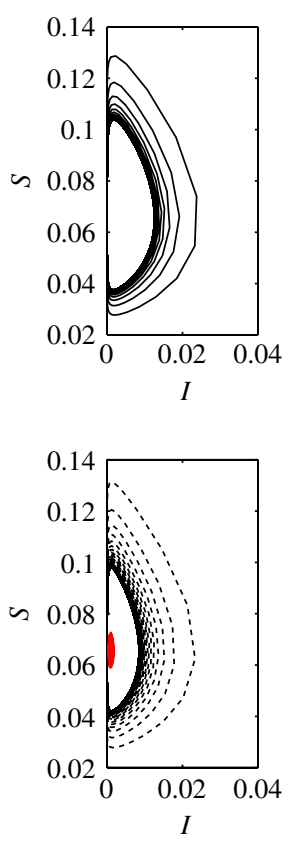
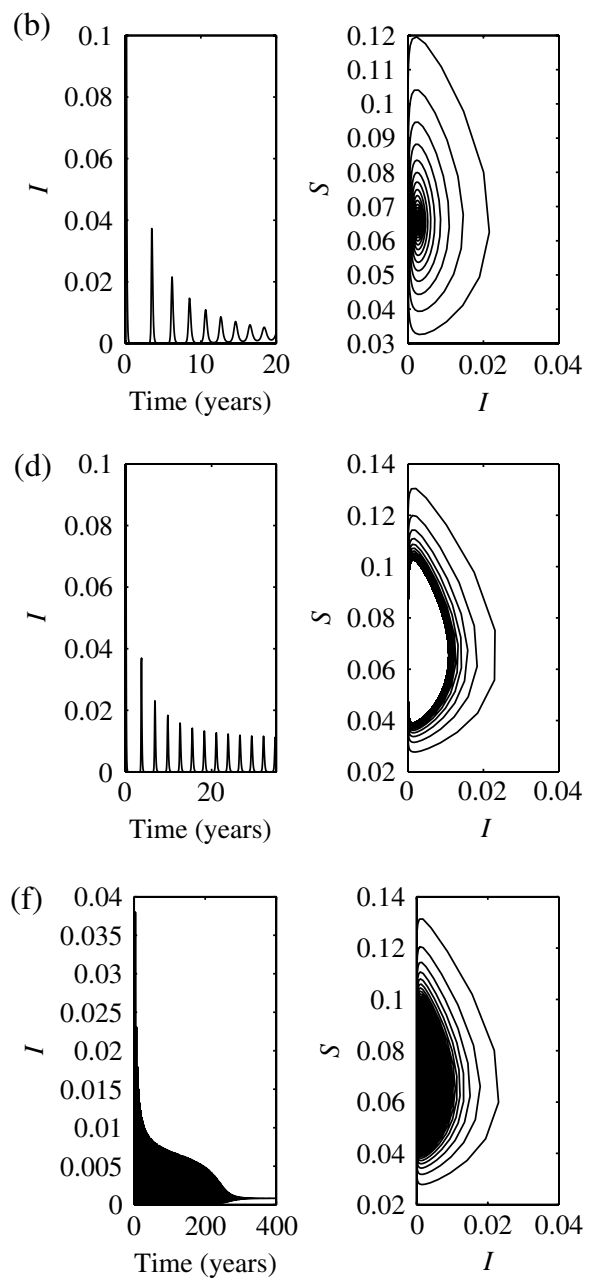

FIGURE 6. Time series plot (left) of the prevalence $I$ and $(S, I$ )-phase plane plot (right) for (a) $v=$ 0, (b) $v=1$, (c) $v=5$, (d) $v=15$, (e) $v=28$, (f) $v=35$. (a) and (b) have $v<v_{\mathrm{H} 1}$, displaying SIRS-like damped oscillatory dynamics. (c) and (d) have $v_{\mathrm{H} 1}<v<v_{\mathrm{H} 2}$, displaying limit cycle dynamics. Note that the amplitude of oscillations is greater for $v=5$, as expected from Figure 5. (e) has $v$ in the region of bistability. Limit cycle dynamics are shown in dashed black (initial conditions $\left.\{S, I, R, W\}_{0}=\{0.99,0.01,0,0\}\right)$ and point attractor dynamics in solid red (initial conditions $\left.\{S, I, R, W\}_{0}=\{0.065,0.002,0.906,0.027\}\right)$. (f) has $v$ larger than the limit point of periodic solutions branch, and the system is SIR-like with damped oscillatory dynamics. (Colour available online.) 


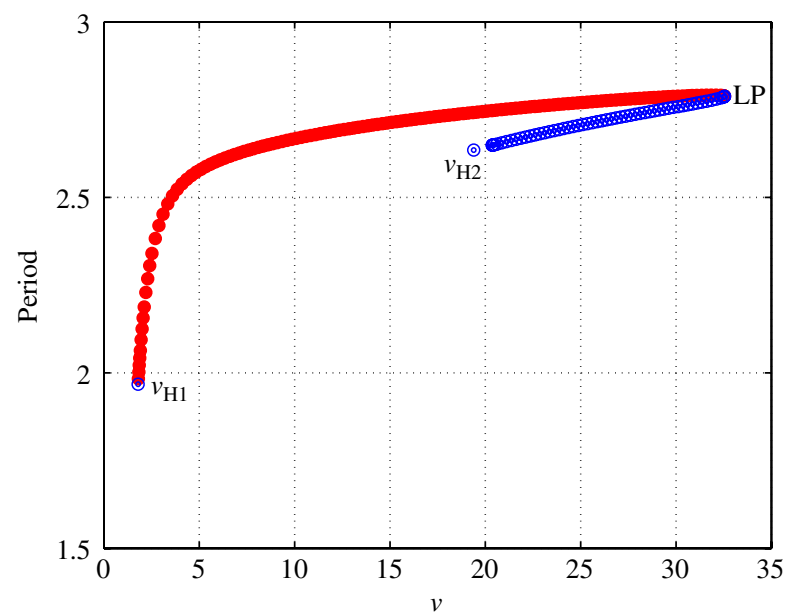

FIGURE 7. Period of the limit cycle as a function of $v$, starting from the Hopf point $v=v_{\mathrm{H} 1}$ (see Figure 5). The stable limit cycle period (solid red circles) extends to the limit point of the periodic solution (LP) at $v \approx 33$, tracking back to $v=v_{\mathrm{H} 2}$ along the unstable limit cycle solution (open blue circles). (Colour available online.)

(a)

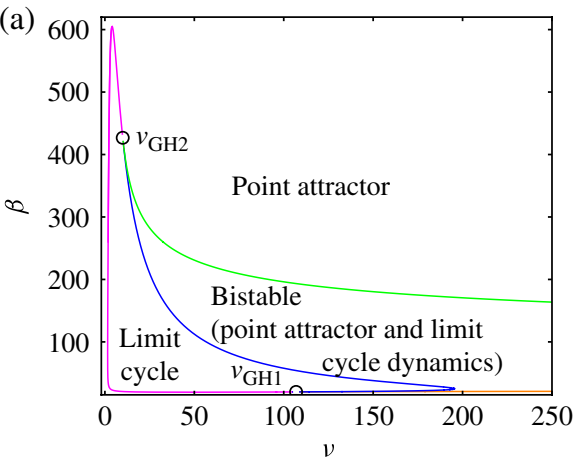

(b) 22.0

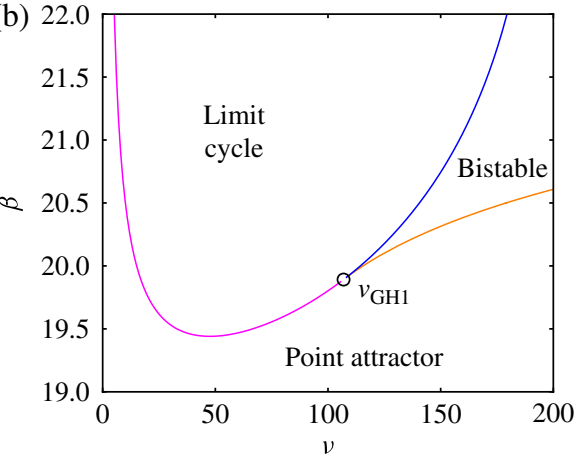

FIGURE 8. Two-parameter bifurcation diagram for the SIRWS system over $R_{0}$ and $v$ (a), with zoom (b), into the small $\beta$ region to show the generalized Hopf point at $(v, \beta) \approx(100,20)$. Noting that $(\gamma+\xi)=17.01$, the disease-free equilibrium loses stability at $\beta=17.01$. For very low $R_{0}>1(17.01<\beta \lesssim 19)$ the system has damped oscillatory dynamics for all values of $v$. A narrow region with a stable limit cycle is then entered until a generalized Hopf point, $v_{\mathrm{GH} 1}$, appears at $\beta \approx 19.7\left(R_{0} \approx 1.15\right)$. As $\beta$ increases, the system has a supercritical Hopf line (magenta), subcritical Hopf line (blue) and limit point of periodic solutions bifurcation line (orange), indicating the existence of point attractor ("small" $v$ ), stable limit cycle ("intermediate" $v$ ) and bistable ("large" $v$ ) dynamics. Of mathematical interest, the green and orange lines converge at $(v, \beta) \approx\left(10^{6}, 30\right)$ (not shown), confirming that the $v \rightarrow \infty$ limit, whereby the system is SIRlike, has point attractor dynamics. As $\beta$ increases further, the region of bistability narrows, extinguished at the generalized Hopf point $v_{\mathrm{GH} 2}$, where the subcritical Hopf line becomes supercritical. (Colour available online.) 
(a)
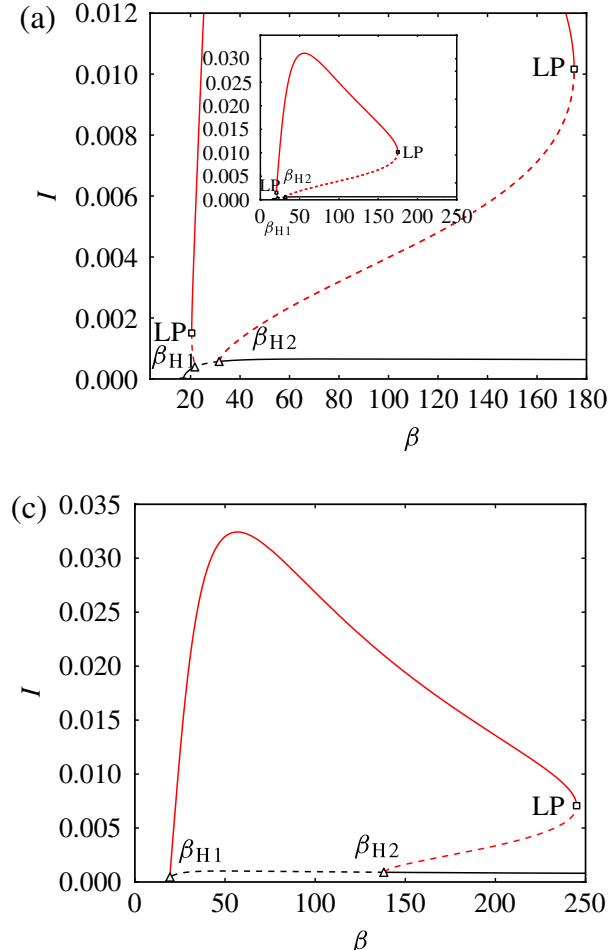

(b)
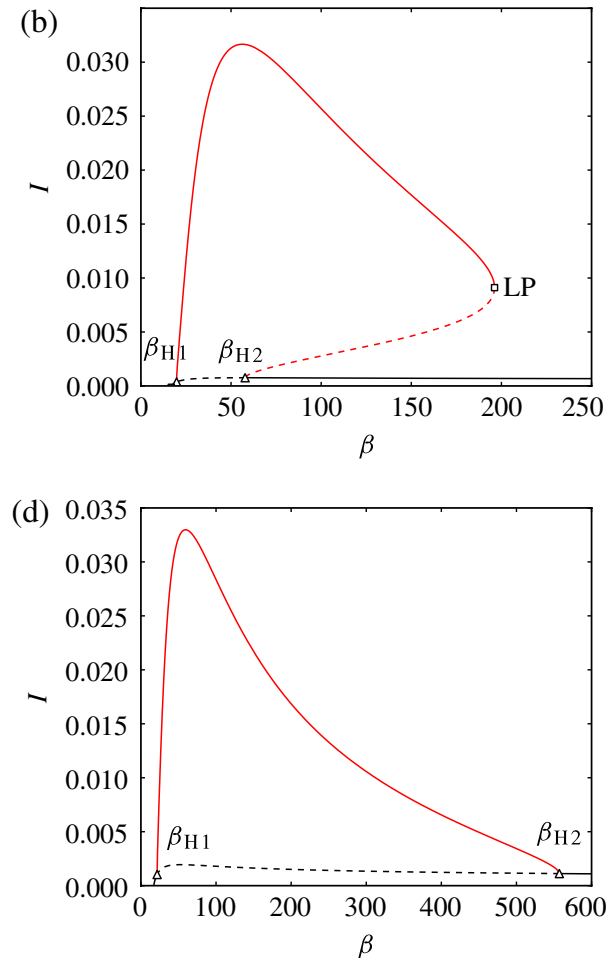

FIgURE 9. Bifurcation diagrams for $\beta$ at different values of $v$, for the diagram shown in Figure 8 , for (a) $v=175$, (b) $v=100$, (c) $v=40$, (d) $v=6$. Continuous and dashed lines represent stable and unstable branches, respectively. The dynamics illustrated in (a) show that both $\beta_{\mathrm{H} 1}$ and $\beta_{\mathrm{H} 2}$ are subcritical, resulting in two regions of bistability (red dotted lines). The inset in (a) shows the extent of the region for periodic orbits originating from the two Hopf points. The limit points (LP) illustrated are points on the orange and green lines, respectively, in Figure 8 . For $v=100<v_{\mathrm{GH} 1}(\mathrm{~b}), \beta_{\mathrm{H} 1}$ changes its stability to supercritical, resulting in only one region of bistability. For $v=40$ (c), the extent of the unstable branch of limit cycles narrows. At $v=6<v_{\mathrm{GH} 2}(\mathrm{~d})$, the second Hopf point $\left(\beta_{\mathrm{H} 2}\right)$ becomes supercritical and no unstable limit cycle is present. (Colour available online.)

While simple expressions for the period of oscillations are not easily formed for the SIRWS model, analytical results are known for the SIRS ( $v \rightarrow 0$ limit) and SIR $(v \rightarrow \infty$ limit) models [10]:

$$
T_{\mathrm{SIRS}}=\frac{4 \pi}{\sqrt{4\left(R_{0}-1\right) \frac{1}{G_{I}} \frac{1}{G_{R}}-\left(\frac{1}{G_{R}}-\frac{1}{A}\right)^{2}}},
$$

where $A=(\kappa+\xi+\gamma) /[(\kappa+\xi)(\beta-\gamma-\xi)]$ is the average age at first infection, $G_{I}=$ $1 /(\gamma+\xi)$ is the average duration of infectiousness and $G_{R}=1 /(\kappa+\xi)$ is the average duration of protection following exposure; and

$$
T_{\mathrm{SIR}}=2 \pi \sqrt{A G},
$$


where $A=1 /\left[\xi\left(R_{0}-1\right)\right]$ is the average age at first infection and $G=1 /(\gamma+\xi)$ is the average duration of infectiousness.

For our choice of parameters, we have $T_{\mathrm{SIRS}}=1.23$ years and $T_{\mathrm{SIR}}=4.03$ years. In comparison, the numerically determined periods from Figure $6(\mathrm{~b})\left(v=1<v_{\mathrm{H} 1}\right.$, SIRSlike) and Figure 6(f) ( $v=35$, greater than the limit point of the periodic solution, SIRlike) are $T_{v=1}=1.83$ years and $T_{v=35}=2.88$ years, respectively. A period of 4 years is achieved for $v=3000$, but we caution that the scale over which the system converges to the $v \rightarrow \infty$ limit is strongly dependent upon the full model parameterization (see Section 2.6).

2.6. The dependence on $\boldsymbol{R}_{\mathbf{0}}$ Finally, we examine how a change in a key biological parameter modifies the characteristic dynamics of the system. We use $R_{0}$ (note that $R_{0}$ is proportional to $\beta$ ) to demonstrate our approach.

Figure 8 shows a two-parameter bifurcation diagram, showing the supercritical (magenta), subcritical (blue) and limit point of periodic solutions bifurcation lines (green, orange). For large $R_{0} \gtrsim 35$ ( $\left.\beta \gtrsim 600\right)$, the system supports only stable point attractor (damped oscillatory) dynamics. As $R_{0}$ is reduced, the system undergoes a Hopf bifurcation, picking up a pair of supercritical Hopf lines (magenta), allowing for stable limit cycle dynamics for a narrow range of boosting values. At the generalized Hopf point $v_{\mathrm{GH} 2}$, the right supercritical Hopf line becomes subcritical (blue), allowing for bistable dynamics for $v$ lying to the right of the subcritical line (blue) and left of the limit point of periodic solutions bifurcation line (green). The range for $v$ in which bistable dynamics are found grows rapidly as $R_{0}$ is further reduced. Note that for very large $v$, the two limit point of periodic solutions bifurcation lines converge.

It is also instructive to consider in more detail how the dynamics of the system change with $\beta$ for fixed $v$. One-parameter bifurcation diagrams in terms of $\beta$ are presented in Figure 9, showing the qualitative changes in the model dynamics for four different fixed values of $v$ (that is, vertical slices through Figure 8). We select $v=175$ lying to the right of the generalized Hopf point $v_{\mathrm{GH} 1} ; v=100$ and $v=40$ lying between the two generalized Hopf points $v_{\mathrm{GH} 1}$ and $v_{\mathrm{GH} 2}$; and $v=6$ lying to the left of $v_{\mathrm{GH} 2}$.

For $v=175$ (Figure 9(a)), the system supports two regions of bistability as both Hopf points are subcritical. The first region is for a narrow range of low values for $\beta$, shown as the dotted line linking (the leftmost) LP and $\beta_{\mathrm{H} 1}$, corresponding to the vertical region between the orange and blue lines in Figure 8(b). The second, separated by a region characterized by limit cycle dynamics, is shown as the dotted line linking (the rightmost) LP and $\beta_{\mathrm{H} 2}$, and corresponds to the region lying between the (upper segment of the) blue line and the green line in Figure 8. Figures 9(b) and (c), each with $v_{\mathrm{GH} 2}<v<v_{\mathrm{GH} 1}$, show dynamics with one region of bistability. Figure $9(\mathrm{~d})$, with $v<v_{\mathrm{GH} 2}$, is characterized by point attractor and stable limit cycle dynamics only.

The epidemiological significance of the results is that for low but plausible values of $R_{0}$ (say, $\beta \lesssim 200$, corresponding to $R_{0} \lesssim 11$ ), the system does not display SIR-like dynamics for biologically plausible values of the boosting. SIRS-like (small $v$ ) point attractor, stable limit cycle and bistable dynamics remain plausible. For higher values 
of $R_{0}$ (here, greater than approximately 25 , namely $\beta \gtrsim 420$ ), the system does not exhibit bistable behaviour. We note that the particular numerical values found here depend upon the values assumed for the life expectancy, infectious period and waning period.

\section{Final remarks}

We have examined the characteristic dynamics of a susceptible-infectiousrecovered-susceptible model with immune boosting. Our findings suggest that with increasing life expectancy the system may naturally transition from one with point attractor dynamics to one characterized by stable limit cycles or even bistable behaviour.

Over biologically plausible ranges for the boosting $v$, we found undamped behaviour, suggesting a role for immune system boosting in the maintenance of nonequilibrium dynamics. Interestingly, we also identified a region of bistability, in which a perturbation to the system, through, say, an intervention measure such as a pulsed vaccination campaign, may be able to transition the system from the stable limit cycle to the point attractor. By reducing the time variation in disease, such an intervention may prove advantageous for both monitoring of disease and further mitigation efforts.

Our system has a number of limitations. Among them, the demographics model is simplistic (but mathematically convenient) and leads to a population age structure more comparable to developing world populations. Comparison of the results to those from a model in which all individuals live to age $1 / \xi$ may be instructive. Similarly, although likely precluding the form of analysis presented here, inclusion of age structure and the mixing between age groups is generally acknowledged to be important when considering the detailed behaviour of particular infectious disease systems.

The model discussed here has previously been introduced in the context of explaining rising pertussis incidence despite high vaccination coverage [11]. That analysis focused on the potential for vaccination to cause transitions from dynamics characterized by a stable point attractor (damped oscillations) to a stable limit cycle (undamped oscillations). Our analysis builds upon this work by showing that within the observable range of life expectancies (50-100 years), diverse dynamics can occur in the absence of vaccination. While life expectancy is relatively easy to identify in a given population, we suggest caution be used in real-world applications, given that transitions appear to occur well within the precision to which other transmission parameters are commonly known.

In conclusion, we hope that our investigations may contribute to the ongoing efforts to better understand the changing (and arguably worsening) epidemiological profile of endemic diseases such as pertussis, for which immunity is not lifelong and immune boosting is thought to play an important role in the maintenance of immunity. We suggest that underlying changes in the demographic state of the population may have 
dramatic influences on the characteristic dynamics of SIRS systems with immune boosting, and caution against attributing changes in the characteristic dynamics to vaccination alone.

\section{References}

[1] R. M Anderson and R. M. May, Infectious diseases of humans: dynamics and control (Oxford University Press, Oxford, 1992).

[2] M. S. Bartlett, "Measles periodicity and community size", J. R. Stat. Soc. A 120 (1957) 48-70; doi: $10.2307 / 2342553$.

[3] C. T. Bauch and D. J. D. Earn, "Transients and attractors in epidemics", Proc. R. Soc. Lond. B 270 (2003) 1573-1578; doi:10.1098/rspb.2003.2410.

[4] P. Van Damme and M. Burgess, "Immunogenicity of a combined diphtheria-tetanus-acellular pertussis vaccine in adults", Vaccine 22 (2004) 305-308; doi:10.1016/j.vaccine.2003.08.012.

[5] E. J. Doedel, "AUTO: a program for the automatic bifurcation analysis of autonomous systems", Congr. Numer. 30 (1981) 265-284.

[6] E. J. Doedel, AUTO-07P: Continuation and bifurcation software for ordinary differential equations, 2007; http://www.ma.hw.ac.uk/ gabriel/auto07/auto.html.

[7] E. J. Doedel, A. R. Champneys, T. F. Fairgrieve, Y. A. Kuznetsov, B. Sandstede and X. Wang, AUTO 97: Continuation and bifurcation software for ordinary differential equations (with Hom Cont), 1997.

[8] D. J. D Earn, P. Rohani, B. M. Bolker and B. T. Grenfell, "A simple model for complex dynamical transitions in epidemics", Science 287 (2000) 667-670; doi:10.1126/science.287.5453.667.

[9] B. Ermentrout, "Simulating, analyzing, and animating dynamical systems: a guide to XPPAUT for researchers and students", Volume 14 of Software, Environments, and Tools (Society for Industrial and Applied Mathematics, Philadelphia, 2002).

[10] M. J. Keeling and P. Rohani, Modeling infectious diseases in humans and animals (Princeton University Press, Princeton, NJ, 2008).

[11] J. S. Lavine, A. A. King and O. N. Bjørnstad, "Natural immune boosting in pertussis dynamics and the potential for long-term vaccine failure", Proc. Natl. Acad. Sci. 108 (2011) 7259-7264; doi:10.1073/pnas.1014394108. 